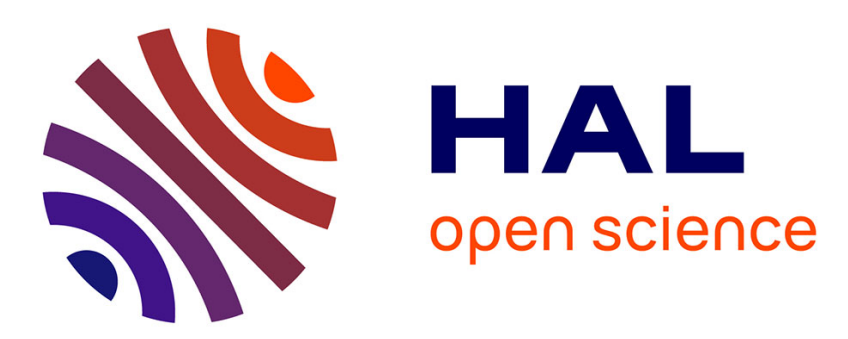

\title{
Design and use of a gradiometer connected rubidium magnetometer
}

C. Osgood

\section{To cite this version:}

C. Osgood. Design and use of a gradiometer connected rubidium magnetometer. Revue de Physique Appliquée, 1970, 5 (1), pp.113-118. 10.1051/rphysap:0197000501011300 . jpa-00243342

\section{HAL Id: jpa-00243342 https://hal.science/jpa-00243342}

Submitted on 1 Jan 1970

HAL is a multi-disciplinary open access archive for the deposit and dissemination of scientific research documents, whether they are published or not. The documents may come from teaching and research institutions in France or abroad, or from public or private research centers.
L'archive ouverte pluridisciplinaire HAL, est destinée au dépôt et à la diffusion de documents scientifiques de niveau recherche, publiés ou non, émanant des établissements d'enseignement et de recherche français ou étrangers, des laboratoires publics ou privés. 


\title{
DESIGN AND USE OF A GRADIOMETER GONNEGTED RUBIDIUM MAGNETOMETER
}

\author{
By C. OSGOOD, \\ University of Exeter, Department of Physics, Devon (Great-Britain).
}

\begin{abstract}
Two rubidium magnetometers are connected by a telephone link and are used to study magnetic field differences between Exeter and Sidmouth (a separation of about 18 miles) in Devon, England. The system has a noise level of less than $50 \mathrm{~m} \gamma$ and is notable for its simplicity. Some advantages of the system are demonstrated by examples of the results obtained, which illustrate coastal and tidal effects in the geomagnetic variations.
\end{abstract}

Introduction. - The original object of this experiment was to study the effects of a coastline on geomagnetic micropulsations. To fulfil this object, an instrument was required which would be capable of measuring magnetic field changes of the order of a few gamma, with an accuracy and stability such that simultaneous measurements at two sites could be reliably compared. It was hoped that this comparison would then yield information on the changes in micropulsations caused by a coastline. It would clearly be advantageous if this comparison could be made automatically, and for this reason a system based on rubidium oscillators was chosen, since the frequency modulated signals could be easily transmitted between observation sites.

The University of Exeter maintains a Geophysical Observatory at Sidmouth (see fig. 1), in Devonshire, England. One oscillator was already working here

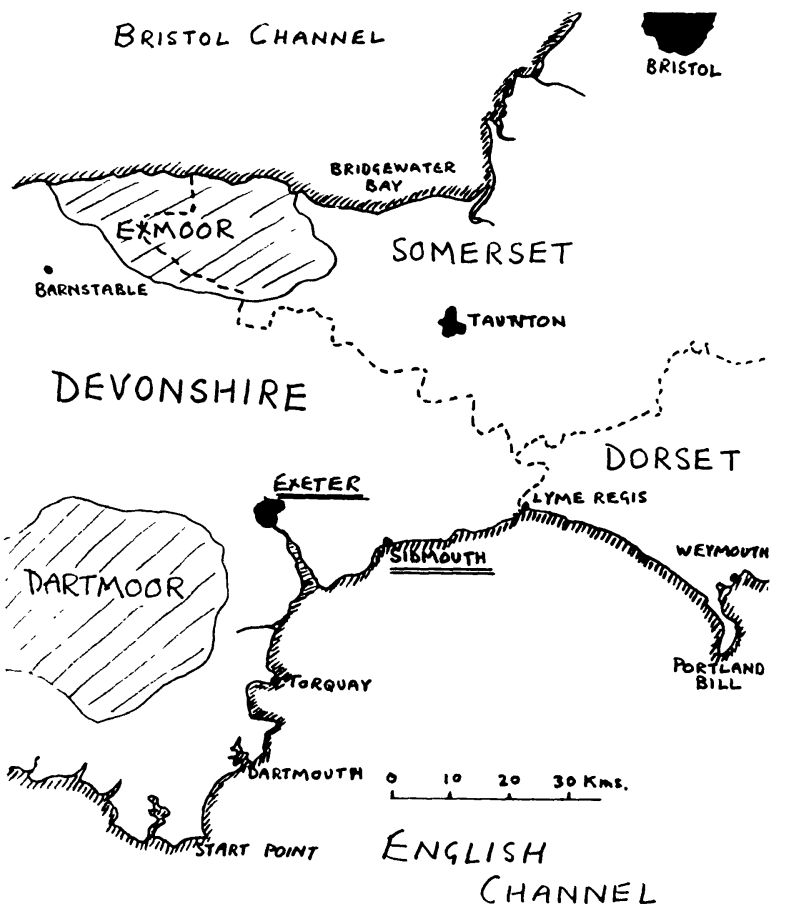

FIG. 1. (about $1 \mathrm{~km}$ from the coast) and the other was placed in the grounds of the University (about $10 \mathrm{~km}$ from the coast). Since the main interest was in micropulsation activity, the output was filtered so that only field changes in the frequency range $5 \mathrm{mHz}$ to $1 \mathrm{~Hz}$ were recorded. However, during 1968, Dr. K. Weekes began to suspect that the variation, of lunar semidiurnal period, which he had found in the magnetic total field data from Sidmouth, had its main origin in the tidal motions of the water in the English Channel. To test this theory an unfiltered output was incorporated in the instrument.

The gradiometer. - The basis of the instrument consists of two Rubidium Oscillators and a Readout Unit made by Varian Magnetometers Ltd. The remainder of the instrument was built by the author. The instrumentation provides six output channels which are a "fast channel" (meaning $5 \mathrm{mHz}-1 \mathrm{~Hz}$ ) and a 'D.C.' channel, that is the unfiltered channel, for each of the three components :

1) Total field at Sidmouth;

2) Gradiometer;

3) Total field at Exeter.

Only the construction of the gradiometer will be described, since the total field channels are very similar - differing only in component values. The Varian Readout Unit provides an audiofrequency output, at about $1.5 \mathrm{kHz}$, which is obtained by mixing the Rubidium Oscillator output with a signal from a crystal oscillator. The natural frequency of a Rubidium Oscillator, at this latitude, is about $220 \mathrm{kHz}$, so that a crystal oscillator frequency of $219 \mathrm{kHz}$ is used. A block diagram of the apparatus si shown in figure 2 .

R.F. Mixer. - The radiofrequency $(220 \mathrm{kHz})$ mixer consists of a field effect transistor which has a double gate. The reference signal, from the crystal oscillator, is applied to one gate and the Rubidium Oscillator signal to the other, with the transistor operating as a common source amplifier. The output is detected with a diode, integrated and then fed back to both gates so that some audioamplification is obtained - the conversion gain being about $6 \mathrm{~dB}$. 


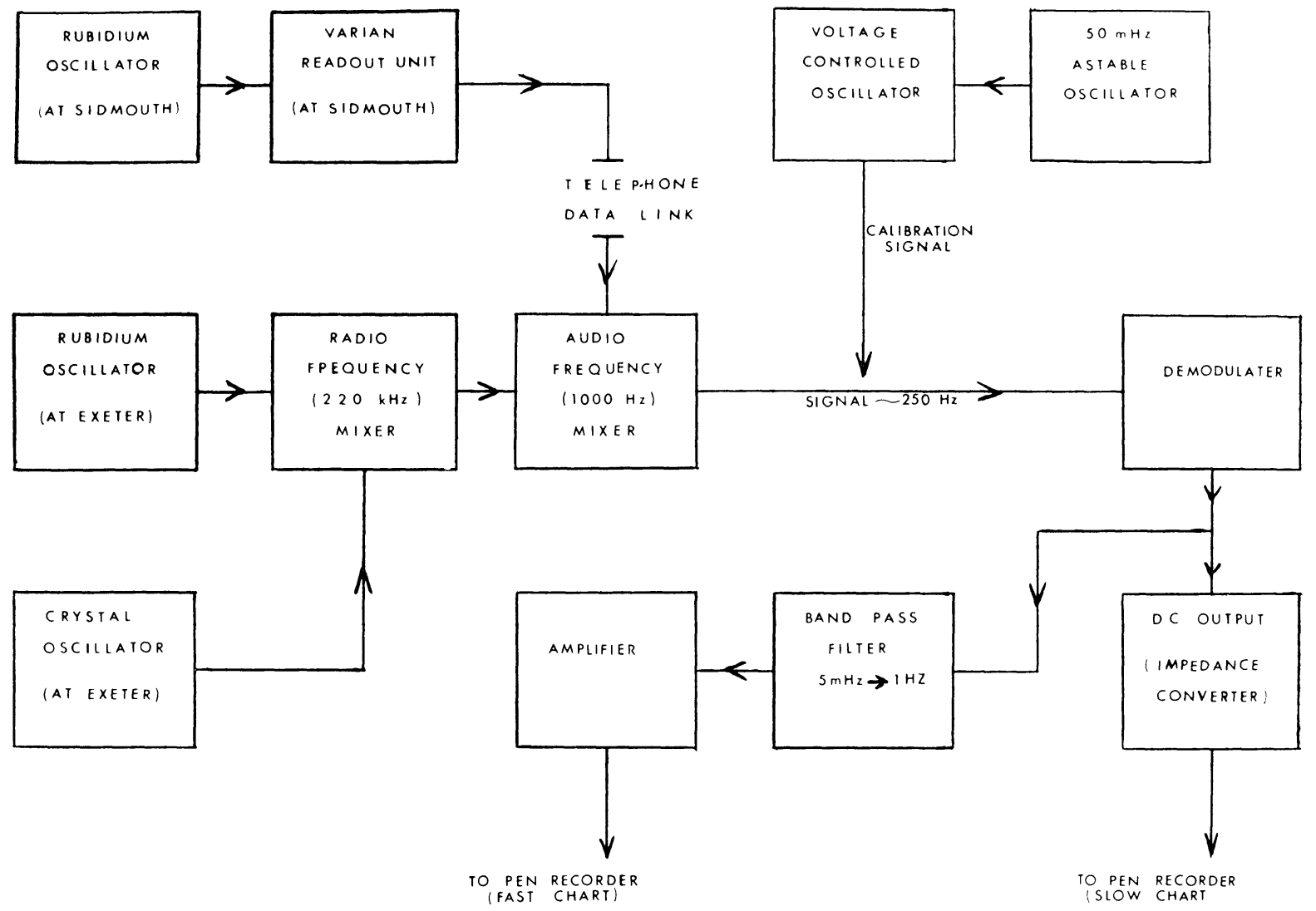

Fig. 2. - Block of the gradiometer.

Since the mixing is carried out at high level, both signals being of the order one volt peak to peak, the type of mixer used is not of great importance. The stability of the crystal oscillator is assured by placing the crystal in an oven, the temperature of which is controlled to within $0.1{ }^{\circ} \mathrm{C}$.

A.F. Mixer. - The audiofrequency from the Varian Readout Unit at Sidmouth is transmitted to Exeter via a telephone data link. The two audiosignals are demodulated separately, to provide the total field outputs, and are also fed into a mixer to provide the gradiometer output. Since the ratio of input frequency to output frequency is only about $4: 1$, it was found that the most efficient mixer was the well-known diode ring. This circuit only provides a small voltage output and it is thus followed by an integrated circuit, used as a limiting amplifier, to provide a square wave output.

The Demodulator. - The signal is then demodulated to give an analogue output suitable for display on a pen-recorder. The demodulator consists of four parts as follows :

1) Schmidt trigger;

2) Monostable multivibrator;

3) Amplifier;

4) Integrator.

The Schmidt trigger is used to provide pulses, with sharp, well-defined edges, to trigger the monostable circuit. This circuit then gives 15 volts pulses of constant width which are then amplified to 48 volts peak to peak. This pulse train is integrated with a time constant of about $0.1 \mathrm{~s}$, to give the demodulated output. The output of the demodulator is linear, to within about $1 \%$ for inputs in the range from $150 \mathrm{~Hz}$ to $500 \mathrm{~Hz}$.

D.G. Output. - This is an impedance converter consisting of an emitter follower, and a complementary stage, with an output impedance of about $300 \mathrm{ohms}$, to feed a pen-recorder. The time constant of the monostable is adjustable so that an output of $0.0643 \mathrm{~V} / \mathrm{Hz}$, corresponding to $10 \gamma$ full scale deflection, can be obtained.

Fast Channel Output. - The output of the demodulator is also taken to a band pass filter with $3 \mathrm{~dB}$ points at $5 \mathrm{mHz}$ and $1 \mathrm{~Hz}$. The output of this filter is then amplified and the gain of the amplifier adjusted so that full scale deflection on the penrecorder corresponds to $2.5 \gamma$ peak to peak.

Calibration Unit. - This consists of two astable multivibrators. The first oscillates at $50 \mathrm{mHz}$ and the square wave output is then used to control the frequency of a second multivibrator. This second multivibrator has a centre frequency of about $250 \mathrm{~Hz}$ and the frequency change is adjusted to correspond to a magnetic field change of $2.5 \gamma$. Thus the calibration unit gives a $2.5 \gamma$ peak to peak, $50 \mathrm{mHz}$ square wave on the pen-recorder chart.

Agauracy And Stability. - Since the mixer stages introduce no appreciable noise the stability of the instrument is dependent almost entirely on the sta- 


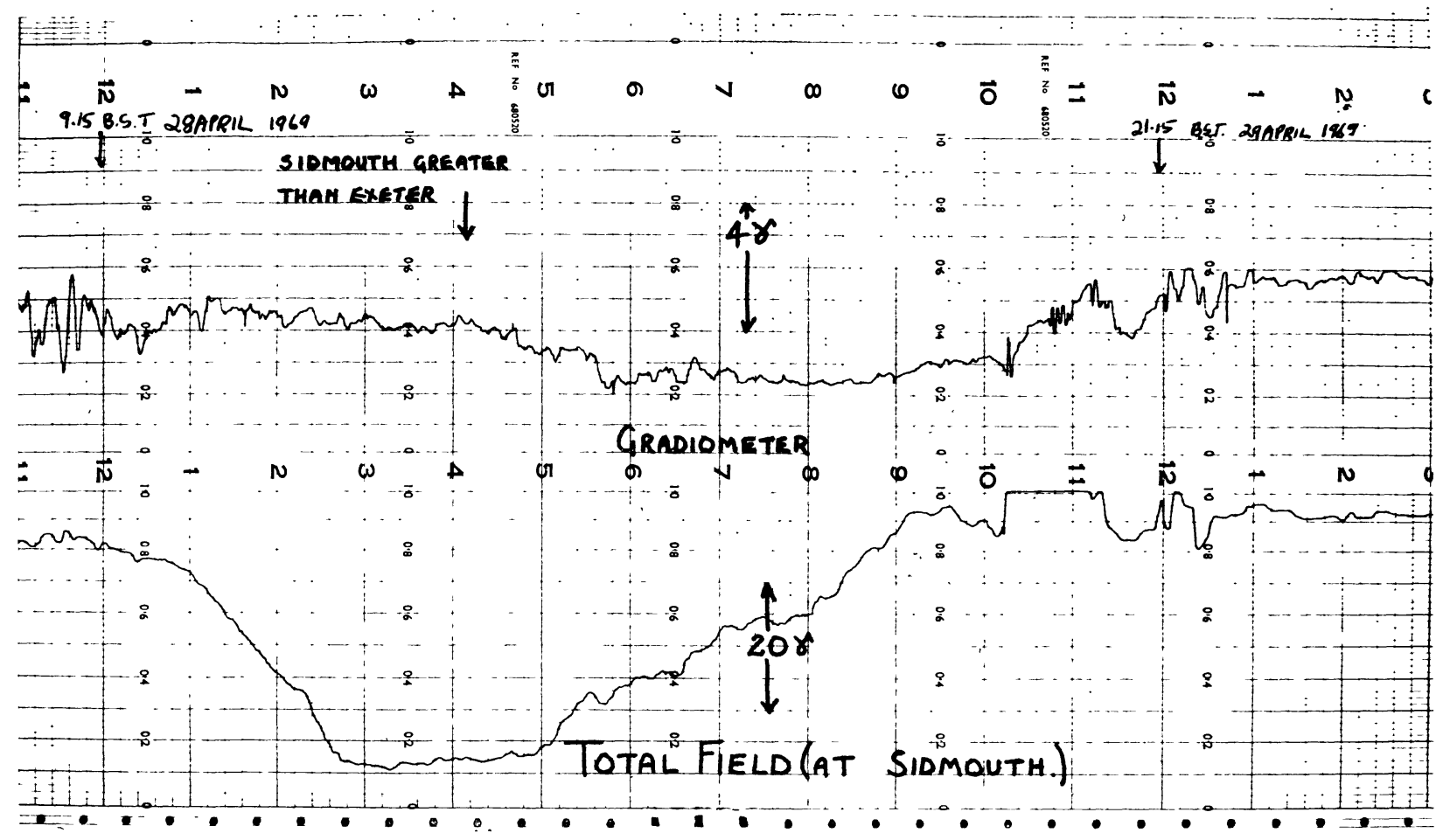

FIG. 3.

bility of the rubidium and crystal oscillators. The Rubidium Oscillator appears to be stable to better than $0.2 \mathrm{~Hz}$ (for a constant magnetic field), and the crystal oscillator has a stability of at least 1 in $10^{7}$. Since the smallest visible output is of the order of $50 \mathrm{~m} \gamma$, the noise level is not easily measurable, but, by inspection of the charts, it is estimated to be less than $50 \mathrm{~m} \gamma$ in the short term, and the drift over one month is less than $0.5 \gamma$.

The demodulator is linear from about $150 \mathrm{~Hz}-$ $500 \mathrm{~Hz}$ and the standing difference frequency is set at about $250 \mathrm{~Hz}$ using biasing magnets, placed near the sensors. The $10 \gamma$ output range is equivalent to $46.66 \mathrm{~Hz}$ and is thus well within the linear range of the demodulator. Using the chart recorder for measurements of linearity, no deviations from linearity were found that were greater than the errors in reading the chart.

It has been found that the $10 \gamma$ range for the D.C. channel is sufficient for most purposes, but that during magnetic storms the instrument is frequently driven off scale. The $2.5 \gamma$ range is not quite sensitive enough for the fast channel and this is shortly to be changed to $1 \gamma$ full scale deflection.

Results. - Although the instrument was originally intended for the study of micropulsations, the most interesting results, so fare, have been found in the D.C. output. This is recorded on paper chart moving at one inch per hour, displaying the three D.C. outputs side by side. The two total field channels are set for $50 \gamma$ full scale deflection. The "fast" outputs are recorded at a chart speed of one inch in 5 minutes and are all $2.5 \gamma$ full scale deflection.

The advantage of the gradiometer is that it eliminates large scale effects, such as the Solar Daily Variation, which are the same at both sensors. This is demonstrated by the section of D.C. chart shown in figure 3.

The lunar tidal effect has two possible origins. 1) Ionospheric-lunar air tides in the ionosphere cause motion of ions leading to current flow, by dynamo action. The magnetic field of this current system is seen on the earth.

2) The Sea-the motions of the sea, moving in the earth's magnetic field cause induced e.m.fs. leading to current flow and thus magnetic field variations.

The characteristics of these two mechanisms are as follows. -1) The ionospheric tidal variation is strongly dependent on the conductivity of the ionosphere and thus should be present only during the solar day. Also it will be a large scale source of magnetic field and would not be expected to give any indication of its presence on the gradiometer, apart from small differences caused by local geological effects.

2) If the effect is due to sea tides, the source of magnetic field will have dimensions of the same order of magnitude as the base-line of the apparatus. Thus significant differences should exist between the amplitudes of the magnetic fields associated with the tides, measured at Sidmouth and Exeter, that is, the tidal effect should be seen by the gradiometer system. Also a semi-diurnal, solar time variation should be seen, since in sea tides the ratio of solar to lunar components is approximately $1: 2$.

One month's gradiometer data was analysed by averaging the results, first in lunar time and then in solar time. The results of these analyses are shown in figure $4 \mathrm{a}$. In figure $4 \mathrm{~b}$, one day's data is plotted with the speed of the tidal stream off the coast of Devonshire.

In figure $4 \mathrm{a}$, we see that the ratio of the lunar to solar night-time amplitudes is of the order of $2: 1$. 

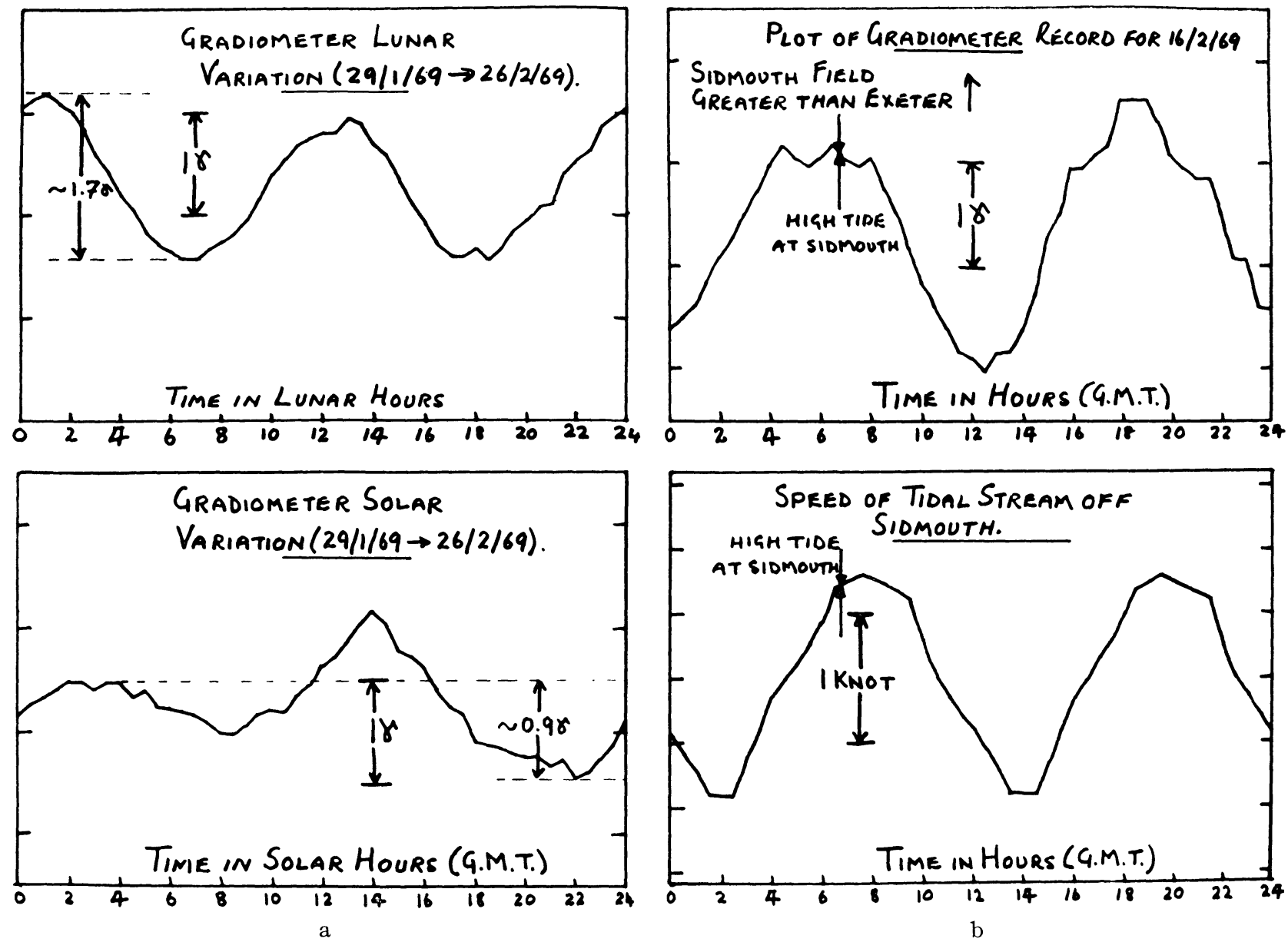

FIG. 4.

a) Mean solar and lunar semidiurnal variations from gradiometer data

b) Comparison of magnetic variations with Tidal Stream Speed in the English Channel off Sidmouth.

The solar daytime amplitude is larger than the nighttime amplitude, for reasons which will be discussed in the next section.

In figure $4 \mathrm{~b}$, the close correlation between the gradiometer measurements and the tidal speed is clearly seen. The evidence of figure 4 shows that the lunar tidal effect, found by Dr. Weekes, has its main origins in the tidal motions of the water in the English Channel. There may be a small component, due to the ionosphere, but this must be small compared to the component from the sea.

The fast channel is also providing interesting results, in that micropulsations have been seen with amplitude differences of as much as $25 \%$ between Sidmouth and Exeter. However, more data and extensive analyses are required before any conclusions can be drawn from this.

Interpretation of results. - The gradiometer provides a very sensitive method for determining the existence of magnetic field differences between the two sensors. However, it must be pointed out that there are some difficulties in interpreting the data obtained. The difficulties encountered with a gradiometer system arise because the sensors used measure only the total field, and the variations of interest are only small perturbations of a large standing magnetic field. Consider the effect of applying a small field $B$ to a sensor where there is also a large standing field $F$. The change measured is given by :

$$
|F+B|-|F|=|F| \cos \theta
$$

where $\theta$ is the angle between $F$ and $B$ and $|B| \ll|F|$. Thus a total field magnetometer effectively measures only the component of the perturbation parallel to the standing field direction.

This statement is true for both sensors of the gradiometer system. Thus if the standing field vectors differ in direction at the two sensors, then an apparent field gradient will be found, even when the applied perturbations are the same for each sensor.

Consider a standing field at Exeter of $F_{\mathrm{E}}$ and at Sidmouth of $F_{\mathrm{s}}$. Let a small field $B$ be applied to each sensor. The measured difference is then given by :

$$
D=\left|F_{\mathrm{E}}+B\right|-\left|F_{\mathrm{S}}+B\right|
$$

where $D$ is the measured difference. Taking the coordinate system shown in figure 5 , where $\theta_{\mathrm{D}}$ is the angle between the main field vectors, both of which lie in the xz-plane, we find that the difference is given by equation (3) :

$$
\begin{aligned}
D & =2|B| \sin \frac{\theta_{D}}{2} \cos \theta \\
& =|B| \theta_{D} \cos \theta
\end{aligned}
$$




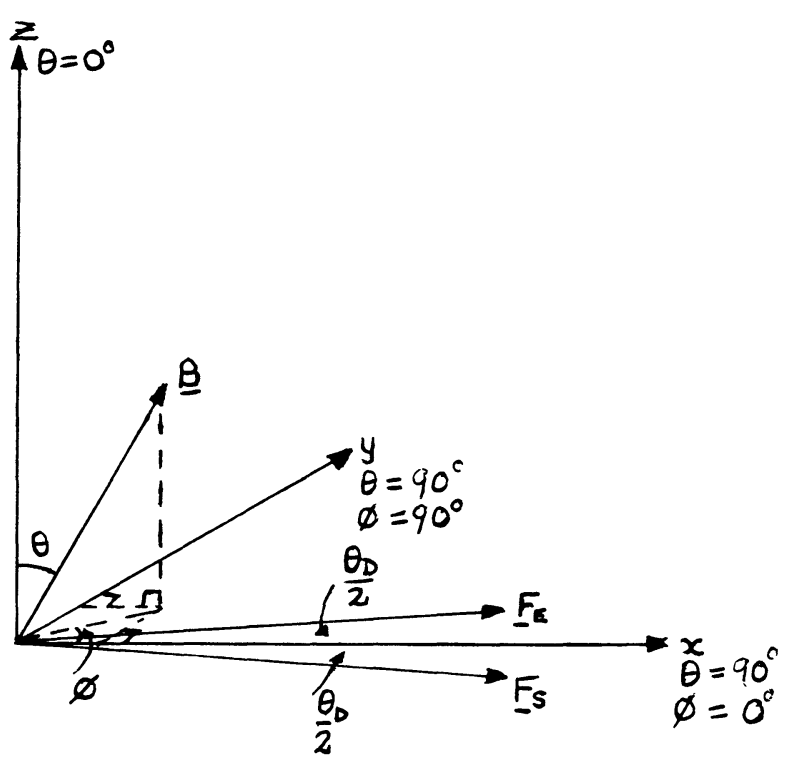

FIG. 5. - Co-ordinate system used for the analysis of the operation of the gradiometer.

where it is assumed that $\theta_{D}$ is small, $|B| \ll\left|F_{\mathrm{S}}\right|$, $\left|F_{\mathrm{E}}\right|=\left|F_{\mathrm{S}}\right|$. If $\left|F_{\mathrm{E}}\right| \neq\left|F_{\mathrm{S}}\right|$ a constant term must be added to equation (3), but this does not effect the present argument as long as the second condition, given above, is met. In figure $6 \mathrm{a}, D$ is plotted as a percentage of the magnitude of $B$ for $\theta_{D}=1^{\circ}$ and $\theta_{D}=5^{\circ}$. If we now consider the anomalously large amplitude of the daytime solar variation, seen in figure 4 a, it can be seen that this could easily be caused by the mechanism discussed in this section. The solar daily variation consists of a flat night-time level which starts to drop at about $06.00 \mathrm{Hrs}$. (L.M.T.), reaches a minimum at about $14.00 \mathrm{Hrs}$. and then rises to the night-time level by about $20.00 \mathrm{Hrs}$. The minimum is typically $30 \gamma \rightarrow 60 \gamma$ below the night-time level. If the "worst case", where $\theta=0^{\circ}$, is considered and $\theta_{D}$ is taken to be $1^{\circ}$, then an apparent difference of about $2 \gamma$ (at $1400 \mathrm{Hrs}$.) could easily be produced. Thus this mechanism can easily account for the anomalous result shown in figure $4 \mathrm{a}$.

An alignment error, of the order of $1^{\circ}$, can easily occur, due to geological effects and the use of biasing magnets. It must be stressed, however, that the error arising from this cause is not generally very large, except when one is trying to measure field differences less than $10 \%$ of the total perturbation field.

To demonstrate this, consider the effect of a field gradient on the system. Let the standing field components at Exeter and Sidmouth be $F_{\mathrm{S}}$ and $F_{\mathrm{E}}$ respectively. Apply a perturbation field $B$ at Exeter and $B+\delta B$ at Sidmouth, where $B$ is parallel to $\delta B$. If this is considered with respect to micropulsations, then typical values for $B$ and $\delta B$ would be $2 \gamma$ and $0.4 \gamma$. The coordinate system shown in figure 5 is used and it can then the shown that the measured difference, $D$, is given by :

$$
\begin{aligned}
D=\left|F_{\mathrm{E}}+B\right|-\mid F_{\mathrm{S}}+B & +\delta B \mid \\
=\left\{\left|F_{\mathrm{E}}\right|-\left|F_{\mathrm{S}}\right|\right\}+\theta_{D}\{ & \left.|B|+\frac{|\delta B|}{2}\right\} \cos \theta \\
& \quad-|\delta B| \sin \theta \cos \Phi
\end{aligned}
$$

where it is assumed that :

a) $\left|F_{\mathrm{E}}\right|-\left|F_{\mathrm{S}}\right| \ll F_{\mathrm{E}}$;

b) $|B| \ll\left|F_{\mathrm{E}}\right|,\left|F_{\mathrm{S}}\right|$;

c) $\theta_{D}$ is small so that $\theta_{D}=\sin \theta_{D}$ and $\cos \theta_{D}=1$.

Thus the perturbation field is measured as a modulation of the standing difference. Since the system cannot distinguish the sign of the difference, it is clear that, to avoid ambiguity, the standing difference must be greater than any changes. If not, partial recti-
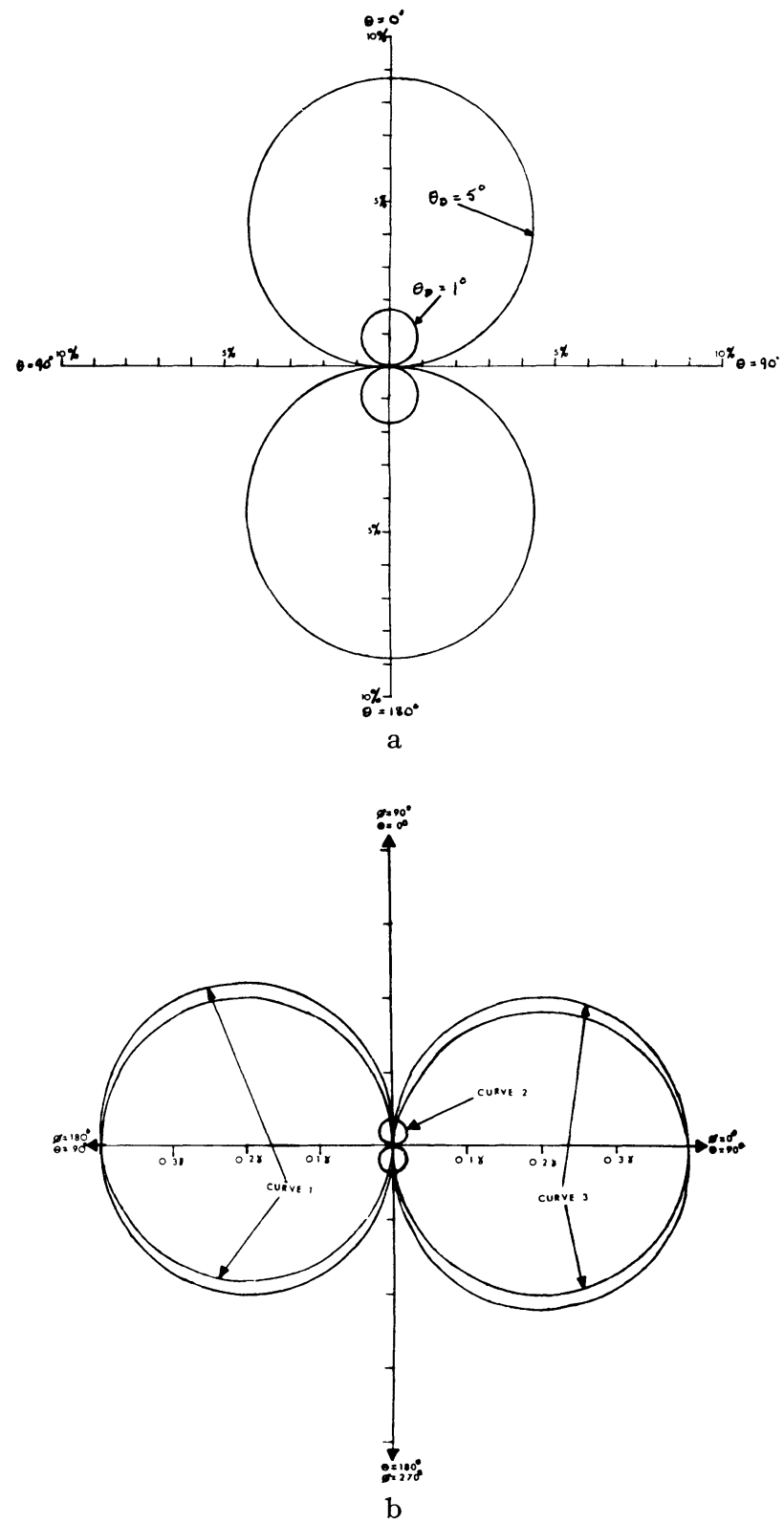

FIG. 6.

a) Polar plot of percentage difference measured when equal perturbations are applied to both sensors, for $\theta_{D}=1^{\circ}$ and $\theta_{D}=5^{\circ}$.

b) Polar plots of measured differences for perturbations of $2 \gamma$ at Exeter and $2.4 \gamma$ at Sidmouth. Curves are plotted for the cases when the perturbation vector lies in one of the coordinate planes i.e. :

Curve 1 : the xz-plane.

Curve $2:$ the yz-plane.

Curve 3 : the xy-plane.

$\theta_{D}$ is taken to be $1^{\circ}$ and the standing difference is zero. 
fication of the output will result, giving rise to apparent doubling of the frequency of the perturbations.

To show the effect of misalignment of the standing field vectors, the standing difference $\left\{\left|F_{\mathrm{E}}\right|-\left|F_{\mathrm{S}}\right|\right\}$ is taken to be zero and the intersections of the function :

$$
D|=| \theta_{D} \cos \theta\left\{|B|+\frac{|\delta B|}{2}\right\}-|\delta B| \sin \theta \cos \Phi \mid
$$

with the coordinate planes, are plotted in figure $6 \mathrm{~b}$.

Curve 1 is for a perturbation in the xz-plane, curve 2 the zy-plane and curve 3 the xy-plane. If $\theta_{D}$ is zero, curves 1 and 3 become identical and curve 2 contracts to a point - the origin.

Thus we see, from equation (4), that the error caused by misalignment of the standing field vectors is given by :

$$
E_{D}=\theta_{D}\left\{|B|+\frac{|\delta B|}{2}\right\} \cos \theta
$$

and the magnitude of this function (i.e. $\left|E_{D}\right|$ ) is curve 2 in figure $6 \mathrm{~b}$. For $\theta_{D}$ equal to $1^{\circ}$ (which is 0.0174 radian), this error can never be more than about $1.7 \%$ of the average amplitude of the perturbation field.

Conclusions. - The instrument is very sensitive and can easily detect magnetic field differences of less than $100 \mathrm{~m} \gamma$, between the two sensors. However, if it is suspected that the standing field directions are different, at the two sensors, then care must be taken in interpreting the results.

For complete studies of the effects of coastlines on natural magnetic phenomena, it will be necessary to use a vector instrument. However, it is felt that the rubidium instrument, with its essential simplicity, is ideally suited for these initial investigations.

I would like to express my thanks to Dr. K. Weekes, and especially to Dr. W. V. G. Rosser, under whose supervision and guidance this work was carried out. 